\title{
Effect of anti-VEGF drugs combined with photodynamic therapy in the treatment of age-related macular degeneration
}

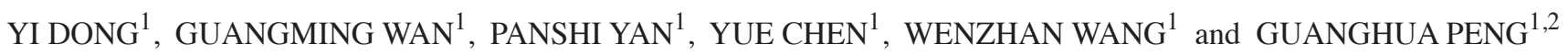 \\ ${ }^{1}$ Department of Ophthalmology, The First Affiliated Hospital of Zhengzhou University, Zhengzhou, Henan 450052; \\ ${ }^{2}$ Chinese PLA General Hospital, Beijing 100853, P.R. China
}

Received July 7, 2016; Accepted November 3, 2016

DOI: $10.3892 /$ etm.2016.3886

\begin{abstract}
We analyzed the effects of anti-vascular endothelial growth factor (VEGF) drugs combined with photodynamic therapy (PDT) in the treatment of age-related macular degeneration (AMD). Ninety-six cases (192 eyes) of AMD were included in this study and randomly divided into the observation group and control group ( $\mathrm{n}=48$ cases per group). The control group was administered the treatment of Lucentis intravitreal injection alone and the observation group was administered Lucentis combined with PDT. The therapeutic effects were compared. The best corrected visual acuity of patients in the two groups increased gradually after treatment. Patients in the observation group had a significantly higher visual acuity when compared to the control group 1 and 6 months post-operation. The differences were statistically significant $(\mathrm{P}<0.05)$. The proportion of patients with vision improvement in the observation group was higher than that in the control group from 1 to 6 months; differences were statistically significant $(\mathrm{P}<0.05)$. Through detection by color Doppler ultrasound within 6 months after treatment, we observed that the peak systolic velocity and arterial end diastolic velocity of retrobulbar optic nerve bitemporal PCA of the observation group were higher than those of the control group. The values of arterial resistance index and pulsatility index of the observation group were lower than those of control group. The differences were statistically significant $(\mathrm{P}<0.05)$. Six months after treatment, the value of central foveal thickness of the observation group was lower than that of the control group, the value of mean sensitivity of visual field parameter $10^{\circ}$ and $4^{\circ}$ was higher in the observation group than in the control group, and the absolute value of mean defects in the observation group were lower than that of the
\end{abstract}

Correspondence to: Dr Guanghua Peng, Department of Ophthalmology, The First Affiliated Hospital of Zhengzhou University, 1 Jianshe East Road, Zhengzhou, Henan 450052, P.R. China

E-mail: peng_guanghua1@163.com

Key words: age-related macular degeneration, anti-vascular endothelial growth factor, drugs, photodynamic therapy control group. In summary, the differences were statistically significant $(\mathrm{P}<0.05)$. Anti-VEGF drugs combined with PDT can optimize the overall vision of patients with AMD, improve hemodynamic parameters and reduce visual field defects.

\section{Introduction}

Age-related macular degeneration (AMD) is the most common cause of blindness in the elderly population (1). Accompanied by retinal pigment epithelial detachment and macular hemorrhage, AMD is mainly caused by regional perfusion disturbance of the central fovea-supplying vessels and metabolite accumulation (2), which seriously affects the quality of life of the elderly population. Many studies worldwide $(3,4)$ have found that vascular endothelial growth factor (VEGF) is directly related to the occurrence of AMD and anti-VEGF drug treatment is an important method for AMD. Anti-VEGF drugs can hinder the progress of AMD, but exert limited effects on optimizing visual function (5).

Photodynamic therapy (PDT) has been proved to be effective in the treatment of neovascular AMD (6), but the expression of angiogenesis factor increased after treatment, which may worsen the recovery of visual acuity (7). A combined treatment of anti-VEGF and PDT can break the limitation of a single treatment and may become the recommended combined treatment method in the future (8). This study uses anti-VEGF drugs combined with PDT to treat patients with AMD with a focus on changes in vision, visual field defects and hemodynamic parameters of optical fundus blood vessels after treatment, and thus, provided a reference basis for clinical treatment.

\section{Materials and methods}

Sample selection. Ninety-six cases (192 eyes) of patients that were diagnosed with AMD at the The First Affiliated Hospital of Zhengzhou University (Henan, China) from January 2013 to January 2016 were selected continuously. Inclusion criteria for the study were: i) Diagnosis of macular degeneration through fundus fluorescein angiography or optical coherence tomography (OCT); ii) age $\geq 60$ years; iii) being administered treatment for the first time; iv) informed consent was obtained from patients and family members; and v) complete clinical data. The exclusion criteria were: History of eye trauma, surgical 
Table I. Comparison of vision improvement within 1 and 6 months after treatment (cases, \%).

\begin{tabular}{lcccccccc}
\hline & & \multicolumn{3}{c}{1 month after treatment } & & \multicolumn{3}{c}{6 months } \\
\cline { 3 - 4 } Groups & Eyes & $\begin{array}{c}\text { Improved } \\
\text { vision }\end{array}$ & $\begin{array}{c}\text { Unchanged } \\
\text { vision }\end{array}$ & $\begin{array}{c}\text { Decreased } \\
\text { vision }\end{array}$ & & $\begin{array}{c}\text { Improved } \\
\text { vision }\end{array}$ & $\begin{array}{c}\text { Unchanged } \\
\text { vision }\end{array}$ & $\begin{array}{c}\text { Decreased } \\
\text { vision }\end{array}$ \\
\hline Observation & 96 & $69(71.9)$ & $20(20.8)$ & $7(7.3)$ & & $82(85.4)$ & $9(9.4)$ & $5(5.2)$ \\
Control & 96 & $50(52.1)$ & $33(34.4)$ & $13(13.5)$ & & $65(67.7)$ & $21(21.9)$ & $10(10.4)$ \\
$\chi^{2}$ test & & & 8.022 & & & & 8.433 & 0.015 \\
P-value & & & 0.018 & & & & \\
\hline
\end{tabular}

history and other retinal diseases. According to therapeutic methods, patients were randomly divided into teh observation and control groups, with 48 cases (96 eyes) in each group. The control group had 25 cases of males and 23 cases of females aged from 62 to 76 years with an average of $69.3 \pm 5.2$ years. There were 31 cases of dry AMD and 17 cases of wet AMD. Twenty cases of patients had comorbidity of hypertension and 10 cases had diabetes. The observation group was composed of 26 males and 22 females aged from 61 to 77 years, with an average of $69.5 \pm 5.6$ years. There were 30 cases of dry AMD and 18 cases of wet AMD. Twenty-two cases of patients had comorbidity with hypertension and 8 cases had diabetes.

Therapeutic methods. The patients in control group were administered anti-VEGF drug treatment, intravitreal injection of Lucentis at a dose of $0.5 \mathrm{mg} / 0.05 \mathrm{ml}$. The injection was repeated once every other month for 3 months.

The patients in the observation group were treated with anti-VEGF drugs combined with PDT, with a dose of $6 \mathrm{mg} / \mathrm{m}^{2}$ verteporfin. Ten minutes after intravenous injection, a laser [with a wavelength of $689 \mathrm{~nm}$, an intensity of $600 \mathrm{~mW} \cdot \mathrm{cm}^{2}$ and an optical density (OD) of $50 \mathrm{~J} / \mathrm{cm}^{2}$ ] was used to directly radiate the retinal lesion area of patients through contact lens for $83 \mathrm{sec}$. The affected eyes were kept out of the sun for 5 days after treatment. Lucentis $(0.5 \mathrm{mg} / 0.05 \mathrm{ml})$ was administered through an intravitreal injection within 3 days after PDT treatment. If bleeding was present, and typical retina angiogenesis and retina hydrops continued to exist in the re-examination after treatment, then intravitreal injection of isodose Lucentis was administered again.

Observation indexes. The best corrected visual acuity was examined before treatment as well as 1 week, 1 and 6 months after treatment. The international standard visual acuity chart was used, according to which improved vision meant that the corrected visual acuity increased by over 2 lines, decreased vision meant that the corrected visual acuity decreased by over 2 lines, and unchanged vision meant that the vision change was within 1 line. For vision $<0.1$, the change in vision of 0.02 was counted as 1 line.

The eye hemodynamic parameters used a color Doppler ultrasound instrument (model iE33; Philips Medical Systems, Andover, MA, USA) with the probe frequency of $11 \mathrm{MHz}$ and sampling volume of 2-3 mm. Patients were placed in a supine position with slightly closed eyes. The probe slightly touched the upper eyelid to conduct horizontal scanning. The dark area
Table II. Comparison of ocular hemodynamic parameter values after treatment.

\begin{tabular}{lcccc}
\hline Groups & $\begin{array}{c}\text { PSV } \\
(\mathrm{cm} / \mathrm{sec})\end{array}$ & $\begin{array}{c}\text { EDV } \\
(\mathrm{cm} / \mathrm{sec})\end{array}$ & RI & PI \\
\hline Observation & $10.81 \pm 1.63$ & $4.83 \pm 0.51$ & $0.54 \pm 0.06$ & $1.12 \pm 0.13$ \\
Control & $8.34 \pm 0.92$ & $3.92 \pm 0.41$ & $0.63 \pm 0.07$ & $1.31 \pm 0.17$ \\
t-test & 4.383 & 4.293 & 5.012 & 5.172 \\
P-value & 0.035 & 0.037 & 0.027 & 0.023 \\
\hline
\end{tabular}

PSV, peak systolic velocity; EDV, end diastolic velocity; RI, arterial resistance index; PI, pulsatility index.

of the optic nerve was displayed on the screen. The pulsed Doppler flow spectrum of retrobulbar optic nerve bitamporal PCA was detected. The peak systolic velocity (PSV) of the ophthalmic artery, arterial end diastolic velocity (EDV), arterial resistance index (RI) and pulsatility index (PI) were recorded. Central foveal thickness (CFT) and visual field parameters were detected by OCT, including the mean sensitivity (MS) within the central vision field scope of $10^{\circ}$ as well as MS and mean defect (MD) within the scope of $4^{\circ}$.

Statistical analysis. The data in this study were analyzed using SPSS 23.0 (SPSS, Inc., Chicago, IL, USA) statistical software. Measurement data are expressed as mean \pm standard deviation after normality test. Independent-samples t-test was applied. Analysis of variance (ANOVA) was applied for repeated measures data. Enumeration data were expressed as ratio and verified by $\chi^{2}$ test. $\mathrm{P}<0.05$ was considered to indicate a statistically significanct difference.

\section{Results}

Comparison of vision improvement. After treatment, the best corrected visual acuity of patients in the two groups increased gradually. Within 1 and 6 months post-operation, the best corrected visual acuity of patients in the observation group was significantly higher than that of the control group. Differences were statistically significant $(\mathrm{P}<0.05)$ (Fig. 1).

The ratio of vision improvement in observation group was higher than that of control group within 1 and 6 months after treatment, and the difference was statistically significant $(\mathrm{P}<0.05)$ (Table I). 
Table III. Comparison of CFT and visual field parameters.

\begin{tabular}{lcccc}
\hline Groups & CFT $(\mu \mathrm{m})$ & $10^{\circ} \mathrm{MS}(\mathrm{dB})$ & $4^{\circ} \mathrm{MS}(\mathrm{dB})$ & $\mathrm{MD}(\mathrm{dB})$ \\
\hline Observation & $261.29 \pm 29.34$ & $24.58 \pm 2.91$ & $23.18 \pm 2.49$ & $-6.73 \pm 0.73$ \\
Control & $295.47 \pm 32.15$ & $21.17 \pm 2.64$ & $20.35 \pm 2.63$ & $-10.14 \pm 0.92$ \\
t-test & 5.342 & 5.495 & 5.124 & 5.372 \\
P-value & 0.020 & 0.018 & 0.024 & 0.019 \\
\hline
\end{tabular}

CFT, central foveal thickness; MS, mean sensitivity; MD, mean defect.

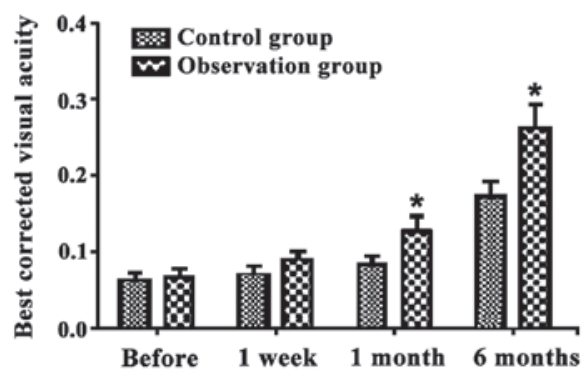

Figure 1. Comparison of best corrected visual acuity before and after treatment. ${ }^{*} \mathrm{P}<0.05$, compared to the control.

Comparison of ocular hemodynamic parameters. Detection through color Doppler ultrasound within 6 months after treatment showed that the PSV and EDV values of the PCA of the observation group were higher than those of the control group. The RI and PI values of the observation group were lower than those of the control group. Differences were statistically significant $(\mathrm{P}<0.05)$ (Table II).

Comparison of CFT and visual field parameters. Within 6 months after treatment, the CFT value of the observation group was lower than that of the control group. In addition, the MS values of visual field parameter $10^{\circ}$ and $4^{\circ}$ of the observation group were higher than those of the control group. Finally, the absolute value of MD in the observation group was lower than that of the control group. Differences were statistically significant $(\mathrm{P}<0.05)$ (Table III).

\section{Discussion}

Lucentis can reduce the leakage of fundus choroidal neovascularization (CNV) and alleviate macular edema, which lead to notable effects in the maintenance of present vision acuity (9). Treatment of neovascular AMD by PDT can retard the central light loss caused by CNV (10).

The most visible clinical manifestation of AMD is the diminution of vision. The best corrected visual acuity is a reliable standard of measuring the eye function and vision of patients. It is generally recognized that the higher the best corrected visual acuity is, the better the holistic vision conditions are (11). This study concluded that the best corrected visual acuity of patients in the two groups increased gradually after treatment, but the observation group had a significantly higher improvement than the control group within 1 and 6 months after operation. The proportion of vision improvement in the observation group was higher than that of the control group within 1 and 6 months, and the differences were statistically significant. Thus, it can be discerned that anti-VEGF drugs combined with PDT lead to significant therapeutic effects. VEGF can cause damage to the function of the blood-retinal barrier, and therefore, directly participates in the occurrence and development of CNV which leads to macular oedema and visual impairment. Intravitreal injection of Lucentis plays a role through the inhibition of angiogenesis by drug use with high local concentration (12). PDT leads to the direct coagulation necrosis of $\mathrm{CNV}$ through the intravenous injection of photosensitive drugs, which gather at a local part of CNV and are activated by low intensity laser (13). Although anti-VEGF drugs can reduce the formed CNV leakage, they lack targeting and cannot block CNV completely (14). PDT can damage the CNV framework that is insensitive to anti-VEGF drugs. Combined with Lucentis, PDT can enlarge the CNV blocking degree and prevent any further damage to vision (15). The simple Lucentis intravitreal injection needs to be repeated many times to achieve an ideal effect, during which it can result in amotio retinae, increase of intra-ocular pressure, traumatic cataracts and other ocular complications (16). After combination therapy, the times of Lucentis injection decreases and the occurrence rate of ocular complications decreases under the premise of inhibiting neovascularization and protecting vision, which may be an important reason for improving holistic therapeutic effects of patients (17).

The root cause of vision improvement of AMD lies in the sealing of CNV and the recovery of haemodynamics of normal ocular fundus (18). The results of this study showed that when detected by color Doppler ultrasound within 6 months after treatment, the PSV and EDV values of PCA of the observation group were higher than those of control group. In addition, the RI and PI values of observation group were lower than those of the control group. The differences were statistically significant. The choriocapillaris of the macular area are dominated by PCA and the regional perfusion disturbance and $\mathrm{CNV}$ formation can cause arteriosclerosis and disorders of the choroidea and retina cycles (19). PSV reflects the degree of angioplerosis and the intensity of blood supply; EDV reflects the blood perfusion state at the distal end of tissue. The higher the PSV and EDV are, the more sufficient the blood supply is (20). RI and PI reflect the blood flow resistance of blood vessels and are negatively related to vascular compliance (21). The results demonstrated that anti-VEGF drugs, combined with PDT, can optimize blood flow state, which is the fundamental mechanism behind vision changes in patients. 
Poor blood supply of the macular area can directly cause macular oedema, the increase of CFT as well as the decreased visual acuity of the macular center and its vicinity (22). The results of this study showed that, within 6 months after treatment, the CFT value of the observation group was lower than that of the control group, the MS values of visual field parameter $10^{\circ}$ and $4^{\circ}$ of the observation group were higher than those of the control group, and the absolute value of MD of the observation group was lower than that of control group. The differences were statistically significant. It showed that the improvement of blood supply to the macular area could alleviate edema of the macular center and decrease CFT value; increased blood supply to the macular area could also directly optimize retinal function in order to improve visual acuity (23).

In conclusion, anti-VEGF drugs, combined with PDT, can optimize the overall vision of patients with AMD, improve hemodynamic parameters and reduce visual field defects, and may therefore, be highly valuable in clinical application.

\section{References}

1. Cho HJ, Kim KM, Kim HS, Lee DW, Kim CG and Kim JW: Response of pigment epithelial detachment to anti-vascular endothelial growth factor treatment in age-related macular degeneration. Am J Ophthalmol 166: 112-119, 2016.

2. Ueda K, Zhao J, Kim HJ and Sparrow JR: Photodegradation of retinal bisretinoids in mouse models and implications for macular degeneration. Proc Natl Acad Sci USA 113: 6904-6909, 2016.

3. Novais EA, Badaró E, Hirai FE, Jorge FA, Leal P, Farah ME and Rodrigues EB: Daily optical coherence tomography examinations after first antivascular endothelial growth factor injections: an interventional case series. J Ophthalmol 2016: 6971831, 2016.

4. Heimes B, Gunnemann F, Ziegler M, Gutfleisch M, Spital G, Pauleikhoff D and Lommatzsch A: Compliance of age related macular degeneration patients undergoing anti-VEGF therapy: analysis and suggestions for improvement. Ophthalmologe 6: Jun 6, 2016 (In German) (Epub ahead of print).

5. Senra H, Ali Z, Balaskas K and Aslam T: Psychological impact of anti-VEGF treatments for wet macular degeneration-a review. Graefes Arch Clin Exp Ophthalmol 4: 12-13, 2016.

6. Arias L, Gómez-Ulla F and Ruiz-Moreno JM: Ranibizumab in monotherapy and combined with photodynamic therapy for retinal angiomatous proliferation. Clin Ophthalmol 10: 861-869, 2016.

7. Wu B, Li J, Lin $\mathrm{H}$ and $\mathrm{Wu} \mathrm{H}$ : Different strategies for the treatment of age-related macular degeneration in China: an economic evaluation. J Ophthalmol 2016: 7689862, 2016.

8. Scherer KM, Bisby RH, Botchway SW and Parker AW: New approaches to photodynamic therapy from type I, II and III to type IV using one or more photons. Anticancer Agents Med Chem 13: 17-18, 2016.

9. Catchpole T, Daniels T, Perkins J and Csaky KG: Method development to quantify Bv8 expression in circulating CD11 $\mathrm{b}^{+}$cells in patients with neovascular age-related macular degeneration (nvAMD) exhibiting anti-VEGF refractoriness. Exp Eye Res 148: 45-51, 2016.
10. Otsuji T, Sho K, Tsumura A, Koike N, Nishimura T and Takahashi K: Three-year results of a modified photodynamic therapy procedure (Ironing PDT) for age-related macular degeneration patients with large lesions. Clin Ophthalmol 10: 431-436, 2016.

11. Arnold JJ: Age-related macular degeneration: anti-vascular endothelial growth factor treatment. BMJ Clin Evid 24: 0701, 2016.

12. Uzun S and Pehlivan E: Comparison of intravitreal aflibercept and ranibizumab injections on subfoveal and peripapillary choroidal thickness in eyes with neovascular age-related macular degeneration. Graefes Arch Clin Exp Ophthalmol 2: $15-16,2016$.

13. Teper SJ, Nowinska A, Pilat J and Wylegala E: Photodynamic therapy in VEGF inhibition non-responders-pharmacogenetic study in age-related macular degeneration assessed with swept-source optical coherence tomography. Photodiagn Photodyn Ther 13: 108-113, 2016.

14. Lee JP, Park JS, Kwon OW, You YS and Kim SH: Management of acute submacular hemorrhage with intravitreal injection of tenecteplase, anti-vascular endothelial growth factor and gas. Korean J Ophthalmol 30: 192-197, 2016.

15. Newman DK: Photodynamic therapy: current role in the treatment of chorioretinal conditions. Eye (Lond) 30: 202-210, 2016.

16. MacDonald DA, Martin J, Muthusamy KK, Luo JK, Pyles E, Rafique A, Huang T, Potocky T, Liu Y, Cao J, et al: Aflibercept exhibits VEGF binding stoichiometry distinct from bevacizumab and does not support formation of immune-like complexes. Angiogenesis 19: 389-406, 2016.

17. Colombeau L, Acherar S, Baros F, Arnoux P, Gazzali AM, Zaghdoudi K, Toussaint M, Vanderesse R and Frochot C: Inorganic nanoparticles for photodynamic therapy. Top Curr Chem 370: 113-134, 2016.

18. Shahin M, Gad MA and Hamza W: Impact of intravitreal triamcinolone acetonide versus intravitreal bevacizumab on retrobulbar hemodynamic in patients with diabetic macular edema. Cutan Ocul Toxicol 33: 49-53, 2014.

19. Toklu Y, Cakmak HB, Raza S, Anayol A, Asik E and Simşek S: Short-term effects of intravitreal bevacizumab [Avastin ${ }^{\circledR}$ ] on retrobulbar hemodynamics in patients with neovascular age-related macular degeneration. Acta Ophthalmol 89: e41-e45, 2011.

20. Mete A, Saygili O, Mete A, Bayram M and Bekir N: Effects of intravitreal bevacizumab (Avastin) therapy on retrobulbar blood flow parameters in patients with neovascular age-related macular degeneration. J Clin Ultrasound 38: 66-70, 2010.

21. Sakalar YB, Senturk S, Yildirim M, Keklikci U, Alakus MF and Unlu K: Evaluation of retrobulbar blood flow by color doppler ultrasonography after intravitreal ranibizumab injection in patients with neovascular age-related macular degeneration. J Clin Ultrasound 41: 32-37, 2013.

22. Koizumi H, Kano M, Yamamoto A, Saito M, Maruko I, Kawasaki R, Sekiryu T, Okada AA and Iida T: Short-term changes in choroidal thickness after aflibercept therapy for neovascular age-related macular degeneration. Am J Ophthalmol 159: 627-633, 2015.

23. Smith RT: New understanding of age-related macular degeneration through quantitative autofluorescence. JAMA Ophthalmol 134: 824-826, 2016. 